\title{
Geometric entanglement of one-dimensional systems: bounds and scalings in the thermodynamic limit
}

\author{
Román Orús ${ }^{1,2}$ and Tzu-Chieh $\mathrm{Wei}^{3}$ \\ ${ }^{1}$ School of Mathematics and Physics, The University of Queensland, QLD 4072, Australia \\ ${ }^{2}$ Max-Planck-Institut für Quantenoptik, Hans-Kopfermann-Straße 1, 85748 Garching, Germany \\ ${ }^{3}$ Department of Physics and Astronomy, University of British Columbia, Vancouver, BC V6T 1Z1, Canada
}

\begin{abstract}
In this paper the geometric entanglement (GE) of systems in one spatial dimension (1D) and in the thermodynamic limit is analyzed focusing on two aspects. First, we reexamine the calculation of the GE for translation-invariant matrix product states (MPSs) in the limit of infinite system size. We obtain a lower bound to the GE which collapses to an equality under certain sufficient conditions that are fulfilled by many physical systems, such as those having unbroken space (P) or space-time (PT) inversion symmetry. Our analysis justifies the validity of several derivations carried out in previous works. Second, we derive scaling laws for the GE per site of infinite-size 1D systems with correlation length $\xi \gg 1$. In the case of MPSs, we combine this with the theory of finite-entanglement scaling, allowing to understand the scaling of the GE per site with the MPS bond dimension at conformally invariant quantum critical points.
\end{abstract}

\section{INTRODUCTION}

Quantum many-body systems in one spatial dimension (1D) have proven relevant in physics. For instance, quantum spin chains have helped to better understand quantum phase transitions [1, 2], the renormalization group [3], quantum state transfer [4], and even the fundamental limitations of classical and quantum computers [5]. In this respect, the advent of White's Density Matrix Renormalization Group (DMRG) [6] was a major breakthrough since it allowed to simulate many of these systems efficiently. At its core, DMRG is based on representing physically-meaningful quantum states in terms of Matrix Product States (MPSs) [7]. Thus, the importance of MPSs can not be overemphasized: they seem to be the correct low-energy description of the relevant quantum states of Nature in 1D [8].

Also, in recent years there has been growing interest in understanding the properties of entanglement in extended systems [9]. While many studies have focused on bipartite measures such as the entanglement entropy and single-copy entanglement [2], there has also been raising interest in investigating the behavior of multipartite quantum correlations. In this respect, among many approaches [9], the so-called geometric entanglement (GE) [10] has been demonstrated to be useful in a variety of situations, including 1D systems and MPSs [11-13], 2D systems [16], and fully-connected symmetric systems [17]. However, and as we shall see here, the study of GE for 1D systems deserves a more detailed analysis in some cases.

The goal of this paper is to analyze in detail two fundamental aspects of the GE of 1D systems in the thermodynamic limit. First, we reexamine the calculation of the GE of MPSs in the limit of infinite size, offering a wide perspective of the problem. As a result, we produce a non-trivial lower bound to the GE. This lower bound collapses to an equality under certain sufficient conditions which we shall make precise later. Moreover, we show that some of these conditions are natural in many physical systems, such as those having unbroken space inversion or parity $(\mathrm{P})$ symmetry or the larger spacetime inversion (PT) symmetry [18]. Second, we derive scaling laws for the GE per site of infinite-size 1D systems with finite but large correlation length. Also, in the case of MPSs we relate these scaling laws with the theory of finite-entanglement scaling [19] and obtain scaling laws with the MPS bond dimension at conformally invariant quantum critical points. In contrast to the diverging behavior of the entanglement entropy of with the subsytem size [2], the GE per site is always bounded, and this gives certain advantage in terms of convergence in numerics.

The remaining of the paper is organized as follows. In Sec. II we review the definition of the geometric measure of entanglement, or in short geometric entanglement (GE). In Sec. III we discuss the bounds on GE per site in the thermodynamic limit. Under certain symmetry, the provided upper bound becomes the exact expression of GE per site. In Sec. IV we investigate the scaling of GE from three different perspectives: (a) finite-correlation length, (b) finite bond-dimension, and (c) finite size. We summarize in Sec. V. We relegate certain detailed discussions on the scaling to Appendix A in order to keep the smooth flow of the main text.

\section{GEOMETRIC ENTANGLEMENT IN A NUTSHELL}

Let us quickly remind the basics of GE. Imagine that we are given a quantum state $|\Psi\rangle$ of $N$ parties belonging to a Hilbert space $\mathcal{H}=\bigotimes_{r=1}^{N} \mathbb{V}^{[r]}$, where $\mathbb{V}^{[r]}$ is the Hilbert space of party $r$. This could be, for instance, the state of a system of quantum spins placed on a lattice where each party is either a single spin or a block of spins. Our aim is to focus on the closest normalized product state of the parties to $|\Psi\rangle$. By "closest" we mean the normalized product state $|\Phi\rangle=\left|\phi^{[1]}\right\rangle \otimes\left|\phi^{[2]}\right\rangle \otimes \cdots \otimes\left|\phi^{[N]}\right\rangle$ 
that minimizes the squared distance ||$|\Phi\rangle-|\Psi\rangle \|^{2}$ between $|\Phi\rangle$ and $|\Psi\rangle$ or, in other words, maximizes the absolute value of their overlap [10], $\Lambda_{\max }(\Psi) \equiv \max _{\Phi}|\langle\Phi \mid \Psi\rangle|$. Notice that finding the maximizing product state in this equation is actually akin to a "mean-field" approximation to $|\Psi\rangle$.

The larger $\Lambda_{\max }$ is, the less entangled is $|\Psi\rangle$. Thus, the closest product state approximation to $|\Psi\rangle$ allows us to quantify its entanglement via the extensive quantity $E(\Psi) \equiv-\log \Lambda_{\max }^{2}(\Psi)[10]$, where we have taken the natural logarithm. As required, $E(\Psi)$ is zero if $|\Psi\rangle$ is unentangled. We can also define the thermodynamic quantity $\mathcal{E}$ and its finite-size version $\mathcal{E}_{N}$ as

$$
\mathcal{E} \equiv \lim _{N \rightarrow \infty} \mathcal{E}_{N}, \quad \mathcal{E}_{N} \equiv N^{-1} E(\Psi)
$$

The quantity $\mathcal{E}$ in the above equation defines the global geometric entanglement per site, or density of global geometric entanglement. This will be the quantity of interest in this paper.

\section{BOUNDS OF GE PER SITE IN THE THERMODYNAMIC LIMIT}

MPSs offer an accurate description of many interesting states of 1D quantum many-body systems. For a system of size $N$ with periodic boundary conditions, these are states defined as

$$
|\Psi\rangle=\sum_{i_{1}, \ldots, i_{N}} \operatorname{tr}\left(A^{[1] i_{1}} \cdots A^{[N] i_{N}}\right)\left|i_{1}, \ldots, i_{N}\right\rangle,
$$

where $A^{[m] i_{m}}$ is a $\chi \times \chi$ matrix at site $m=1, \ldots, N$ for each $i_{m}=1, \ldots, d_{m}$, which labels a local basis of the Hilbert space of dimension $d_{m}$ at site $m$. Parameter $\chi$ is called the bond dimension of the MPS. It is known that any spin state can be written as a MPS, albeit the bond dimension may need to increase with the system size [5]. Therefore, MPS are a good variational family of states to approximate any state, and in particular, ground states of Hamiltonians with local interactions. If the system is invariant under translations of one site (TI) [26], then we can assume to have the same matrices at every site, $A^{[m] i_{m}}=A^{i_{m}} \forall m$. This allows us to take the thermodynamic (infinite-size) limit of an MPS with TI just by considering the same matrices $A^{i_{m}}$ at all the infinitelymany sites $m=1, \ldots, \infty$ (see e.g. Refs. [20]).

Let us now discuss the calculation of the GE of an MPS in the thermodynamic limit. One important assumption that we make now is that the closest product state $|\Phi\rangle$ can be taken to be a product of identical local states, that is, it fulfills TI: $|\Phi\rangle=|\phi\rangle^{\otimes \infty}$. This happens to be a good choice for e.g. the ground states of the transverse-field $\mathrm{XY}$ spin chain, where this ansatz has been verified numerically [11]. For permutation invariant states, this ansatz has been proven correct [17]. However, we caution that sometimes this assumption does not hold. For instance, the state $|\Psi\rangle=2^{-1 / 2}(|0101 \ldots\rangle+|1010 \ldots\rangle)$ is manifestly
TI, whereas its closest product states (e.g. $|0101 \ldots\rangle$ or $|1010 \ldots\rangle)$ are not. In such cases a modified product state ansatz with alternating periodicity (e.g. TI every 2 sites) is the proper choice, as has been verified numerically [1214, 21]. Moreover, one can instead consider GE defined with respect to block product states, and as long as the size of a block is chosen to be a multiple of possible periods of the possible symmetry-breaking states, such translation invariant ansatz is expected to hold.

Under the above assumptions, the overlap between an infinite MPS $|\Psi\rangle$ and a product state $|\Phi\rangle$ reads

$$
\langle\Phi \mid \Psi\rangle=\lim _{N \rightarrow \infty} \operatorname{tr}\left(B_{\phi}^{N}\right),
$$

where $B_{\phi}$ is a $\chi \times \chi$ transfer matrix defined as $B_{\phi} \equiv$ $\sum_{i} A^{i}\langle\phi \mid i\rangle$. Let us call $\lambda_{\phi}^{\alpha}$ the eigenvalues of matrix $B_{\phi}$ (where $\alpha$ labels the different eigenvalues). Then, we have that $\operatorname{tr}\left(B_{\phi}^{N}\right)=\sum_{\alpha}\left(\lambda_{\phi}^{\alpha}\right)^{N} \sim k\left(\lambda_{\phi}^{1}\right)^{N}$, where the last approximation is valid in the limit $N \rightarrow \infty$ and where we assume $\lambda_{\phi}^{1}$ to be the eigenvalue of largest absolute value and $k$ its total degeneracy.

The absolute value of the eigenvalue $\lambda_{\phi}^{1}$ is also known as the spectral radius of matrix $B_{\phi}, \rho_{\phi} \equiv\left|\lambda_{\phi}^{1}\right|$. Another important quantity is the numerical radius of $B_{\phi}$, defined as $w_{\phi} \equiv \max _{\vec{r}}\left|\vec{r}^{\dagger} B_{\phi} \vec{r}\right|$, where $\|\vec{r}\|=1$. A crucial property is that the numerical radius of a matrix upper bounds its spectral radius [23],

$$
\rho_{\phi} \leq w_{\phi}
$$

The above expression turns into an equality if (but not only if) matrix $B_{\phi}$ is diagonalizable.

Our bound for the GE is based on considering $w_{\phi}$ instead of $\rho_{\phi}$ as the relevant dominant scale in the thermodynamic limit for the overlap in Eq. (3). As the latter is not larger than the former, we have specifically that the maximum overlap over product states is bounded as

$$
\Lambda_{\max }(\Psi) \sim \max _{\phi}\left(\rho_{\phi}^{N}\right) \leq \max _{\phi}\left(w_{\phi}^{N}\right)=\left(\max _{\phi}\left(w_{\phi}\right)\right)^{N}
$$

where again the first approximation is valid when $N$ tends to infinity (and up to an irrelevant degeneracy factor independent of $N$ ). Therefore, in the thermodynamic limit the density of geometric entanglement $\mathcal{E}$ in Eq. (1) obeys the bound $\mathcal{E} \geq-2 \log \left(\max _{\phi} \max _{\vec{r}}\left|\vec{r}^{\dagger} B_{\phi} \vec{r}\right|\right)$. The advantage of the latter expression is that it is variational. Furthermore, the double maximization in the right hand side of this equation can be further simplified under the assumption that both maximizations commute $[12,15]$. In such a case, the maximization over $|\phi\rangle$ can be done straightforwardly and we obtain

$$
\mathcal{E} \geq-2 \log \left(\max _{\vec{r}}\left|\left(\vec{r} \otimes \vec{r}^{*}\right)^{\dagger} E\left(\vec{r} \otimes \vec{r}^{*}\right)\right|\right),
$$

where $E$ is the zero-dimensional $\chi^{2} \times \chi^{2}$ MPS transfer matrix $E=\sum_{i} A^{i} \otimes\left(A^{i}\right)^{*}$. The above equation is our main lower bound on the density of GE. 
Let us now discuss the result in Eq. (6). To start with, notice that if the MPS $|\Psi\rangle$ is such that $B_{\phi}$ is always diagonalizable $\forall|\phi\rangle$, then the spectral radius $\rho_{\phi}$ and the numerical radius $w_{\phi}$ coincide and Eq. (6) turns into an equality for the density of GE. Notice also that this is a sufficient condition, but not necessary. In fact, the "equality" version of Eq. (6) was originally derived in the second paper of Ref. [12], where certain assumptions guaranteed the diagonalizability of $B_{\phi}$ for any $|\phi\rangle$. More specifically, it was the (unbroken) symmetry of the physical system under space inversion (P symmetry) the key property that guaranteed this diagonalizability. In order to see this, consider a translation invariant MPS state

$$
|\Psi\rangle=\sum_{i_{1}, \ldots, i_{N}} \operatorname{tr}\left(A^{i_{1}} \cdots A^{i_{N}}\right)\left|i_{1}, \ldots, i_{N}\right\rangle,
$$

and its mirror image state (by reversing the ordering in a $N$-spin basis state without changing the amplitude)

$$
\begin{aligned}
& \left|\Psi^{\prime}\right\rangle=\sum_{i_{1}, \ldots, i_{N}} \operatorname{tr}\left(A^{i_{1}} A^{i_{2}} \cdots A^{i_{N}}\right)\left|i_{N}, i_{N-1}, \ldots, i_{1}\right\rangle \\
= & \sum_{i_{1}, \ldots, i_{N}} \operatorname{tr}\left(\left(A^{i_{N}}\right)^{T} \cdots\left(A^{i_{2}}\right)^{T}\left(A^{i_{1}}\right)^{T}\right)\left|i_{N}, i_{N-1}, \ldots, i_{1}\right\rangle .
\end{aligned}
$$

In order for $|\Psi\rangle=\left|\Psi^{\prime}\right\rangle$, it is sufficient to have $\left(A^{s}\right)^{T}=$ $U A^{s} U^{-1}$, for any invertible $U[24,25]$. Thus, $\left(A^{s}\right)^{T}=A^{s}$ is a sufficient condition for the space inversion or parity (P) symmetry. Such a condition guarantees that any $B_{\phi}$ is automatically symmetric, and therefore always diagonalizable by some orthogonal transformation. $\mathrm{P}$ (or the larger PT) symmetry of the physical system is thus a sufficient condition for any matrix $B_{\phi}$ to be diagonalizable, which in turn is a sufficient condition for Eq. (6) to collapse to an equality. We stress, though, that a matrix $B_{\phi}$ may still be diagonalizable even if the MPS does not have $\mathrm{P}$ (nor $\mathrm{PT}$ ) symmetry.

The general case may be more intricate. To see this, notice that any matrix $B_{\phi}$ (diagonalizable or not) can be written as $B_{\phi}=P_{\phi}^{-1} J_{\phi} P_{\phi}$, where $J_{\phi}$ is the Jordan normal form of $B_{\phi}$ [23]. Matrix $J_{\phi}$ is a direct sum of Jordan blocks, namely $J_{\phi}=\bigoplus_{\alpha} J_{\phi}^{\alpha}$ with $\left(J_{\phi}^{\alpha}\right)_{l, m}=$ $\lambda_{\phi}^{\alpha} \delta_{l, m}+\delta_{l-1, m}, \lambda_{\phi}^{\alpha}$ being the eigenvalues of $B_{\phi}$, and $l, m=1, \ldots, q_{\alpha}$ with $q_{\alpha}$ the size of the Jordan block $\alpha$. An important theorem in linear algebra states that a matrix is diagonalizable if and only if $q_{\alpha}=1 \forall \alpha$, that is, all Jordan blocks are trivial. Thus, the appearance of nontrivial Jordan blocks in the Jordan normal form of $B_{\phi}$ makes it non-diagonalizable, which in turn implies that Eq. (6) may need to remain as a lower bound and not collapse to an equality, depending on the properties of the Jordan blocks in $J_{\phi}$. In such cases, the true value of the GE must be computed using the left hand side of Eq. (5) and maximizing the spectral radius $\rho_{\phi}$ over $|\phi\rangle$, which may be non-trivial. For instance, consider the case of the non-diagonalizable matrix

$$
B_{\phi}=\left(\begin{array}{ccc}
\lambda_{\phi}^{1} & 0 & 0 \\
0 & \lambda_{\phi}^{2} & 1 \\
0 & 0 & \lambda_{\phi}^{2}
\end{array}\right)
$$

which is already in Jordan normal form and such that its eigenvalues satisfy $\lambda_{\phi}^{1} \geq \lambda_{\phi}^{2}$. For simplicity let us assume that these eigenvalues are real and positive. It is easy to check that the spectral radius of this matrix is given by $\rho_{\phi}=\lambda_{\phi}^{1}$, whereas the numerical radius is $w_{\phi}=\max \left(\lambda_{\phi}^{1}, \lambda_{\phi}^{2}+1 / 2\right)$. According to this, we have that for $\lambda_{\phi}^{1} \geq \lambda_{\phi}^{2}+1 / 2$ the numerical and spectral radius coincide and then Eq. (6) collapses to an equality if such a behavior is found for any state $|\phi\rangle$, whereas for $\lambda_{\phi}^{1}<$ $\lambda_{\phi}^{2}+1 / 2$ both radius are different and therefore Eq. (6) must remain as a lower bound.

\section{SCALINGS OF GE PER SITE}

In this section we investigate the scaling of GE from three different perspectives: (a) finite-correlation length, (b) finite bond-dimension, and (c) finite size.

\section{A. Finite- $\xi$ scaling of GE per site}

The GE also obeys precise scaling relations (see e.g. Ref. [12]). We now focus on analyzing the scaling of the density of GE in Eq. (1) as a function of the correlation length $\xi$ for infinite-size 1D systems, where we assume the correlation length to be large. Generally speaking, since the GE per site is a density quantity (similar to the free energy density) the singular part of GE near criticality is expected to behave as

$$
\mathcal{E}(\xi)-\mathcal{E}(\xi=\infty) \sim\left(b+b^{\prime} \log \xi+\cdots\right) / \xi^{d},
$$

where $d$ is the spatial dimension, and $b, b^{\prime}$ modeldependent coefficients. In this expression, we have included a first non-trivial logarithmic correction and assumed that the omitted parts are less singular. In order to assess the validity of Eq. (9) we consider some analytical examples, namely (i) the case of MPSs for which Eq. (6) turns into an equality, and (ii) the quantum XY model for spin $1 / 2$ in the Ising and XX regimes.

Let us then start with the case of MPSs. Under certain assumptions, it is possible to find the corrections due to finite correlation length for the GE per site of an MPS for which the equality version of Eq. (6) holds. First, let us assume that the MPS transfer matrix $E$ obeys an spectral decomposition $E=\sum_{\alpha=1}^{\chi^{2}} \mu^{\alpha} \vec{R}^{\alpha} \vec{L}^{\alpha \dagger}$, where $\vec{R}^{\alpha}$ and $\vec{L}^{\alpha}$ are respectively the $\alpha$ th right and left eigenvectors of $E$ with eigenvalue $\mu^{\alpha}$. If the eigenvalues $\mu^{\alpha}$ are different and rapidly decaying, then this spectral decomposition can be approximated by $E \sim \mu^{1}\left(\vec{R}^{1} \vec{L}^{1 \dagger}+\left(\mu^{2} / \mu^{1}\right) \vec{R}^{2} \vec{L}^{2 \dagger}\right)$, and therefore $\mathcal{E} \sim-2 \log \left|p+\left(\mu^{2} / \mu^{1}\right) q\right|$, with $p$ and $q$ some given coefficients. At this point we recall the standard definition of the correlation length of an MPS, $\xi \equiv-1 / \log \left|\mu^{2} / \mu^{1}\right|$. Using this, together with the assumption $\xi \gg 1$, it is not difficult to arrive at the expression $\mathcal{E}(\xi) \sim a+b / \xi$, with $a=-2 \log |p+q|$ and 
$b=2 q /|p+q|$. We see thus that an MPS is in principle capable of handling $O(1 / \xi)$ corrections. Nevertheless, alternative scaling relations may also hold for MPSs if some of the considered assumptions break down. For instance, it could be the case that the eigenvalues of the MPS transfer matrix $E$ are not rapidly decaying, and therefore $\mathcal{E} \sim-2 \log \left|\sum_{\alpha=1}^{\chi^{2}} \mu^{\alpha} q^{\alpha}\right|$ for the GE (where $q^{\alpha}$ are some coefficients). In principle, this more general expression could account for all the terms in the general scaling law from Eq. (9).

Next, we consider the case of the 1D XY model for spin $1 / 2$, defined by the Hamiltonian

$H_{X Y}=-\sum_{i}\left(\frac{1+r}{2} \sigma_{x}^{[i]} \sigma_{x}^{[i+1]}+\frac{1-r}{2} \sigma_{y}^{[i]} \sigma_{y}^{[i+1]}+h \sigma_{z}^{[i]}\right)$

where $h$ is the magnetic field, $r$ is the anisotropy parameter, and $\sigma_{\alpha}^{[i]}$ is the $\alpha$ th Pauli matrix at site $i$. For $0<r \leq 1$ the model belongs to the universality class of the 1D quantum Ising model (central charge $c=1 / 2$ ). In this regime, in Ref. [11] it was proven that

$$
\left.\frac{\partial \mathcal{E}}{\partial h}\right|_{r \neq 0} \sim-\frac{1}{2 \pi r \log 2} \log |h-1|, \text { for }|h-1| \ll 1 .
$$

Using the above equation together with the known result $\xi=1 /|h-1|$, we can obtain the dependence of $\mathcal{E}$ on $\xi$. Integrating the expression in Eq. (11) we obtain

$$
\mathcal{E}(\xi, r \neq 0) \sim a+\left(b+b^{\prime} \log \xi\right) / \xi,
$$

for $\xi \gg 1$ and where $a, b$ and $b^{\prime}$ are some coefficients independent of $\xi$. Notice that this is the same type of scaling as in Eq. (9). Also, for $r=0$ the model belongs to the universality class of the $1 \mathrm{D}$ quantum XX model (central charge $c=1$ ). In this case, and near $h=1^{-}$, the GE per site was shown [11] to behave as $\mathcal{E}(\xi, r=$ $0) \sim 1 / \xi$, where $\xi \sim|1-h|^{-1 / 2}$. It should be pointed out that although the XX model near this transition is scale invariant, it is not conformally invariant. However, near $h=0$ we have seen numerically that a law like the one in Eq. (12) seems to emerge also for the XX model (see Appendix). Remarkably, we see that all these scaling behaviors accommodate as well in the law hinted in Eq. (9).

\section{B. Finite- $\chi$ scaling of GE per site}

An important fact is that the finite- $\xi$ scalings considered here can be combined with the theory of finiteentanglement scaling [19], in turn allowing to understand the finite- $\chi$ scaling of the GE per site of an MPS with bond dimension $\chi$. In contrast to the diverging behavior of the entanglement entropy of with the subsytem size [2], the GE per site is always bounded above by $\log _{2} d$ ebits, where $d$ is the local Hilbert space dimension, and this gives certain advantage in terms of convergence in numerics. Knowing that close to a conformally-invariant (a)

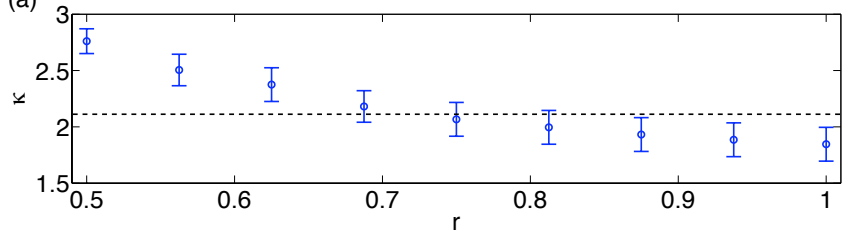

(b)

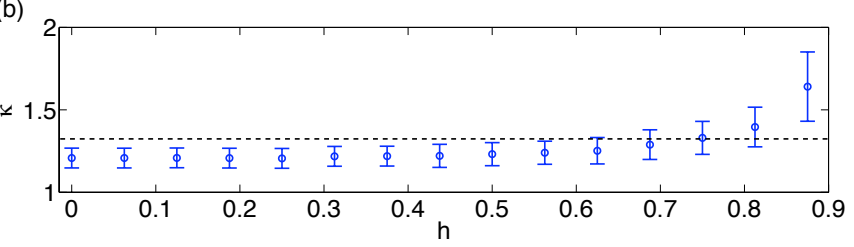

FIG. 1: Exponent $\kappa$ as a function of (a) $r$ for the XY model with $h=1$, and (b) $h$ for the XX model. The values and errorbars correspond to those in tables I and II. Dotted lines correspond to the averages over the studied interval, $\kappa \sim 2.1$ for (a) and $\kappa \sim 1.3$ for (b).

quantum critical point the correlation length obeys the relation $\xi \sim \chi^{\kappa}$ with $\kappa=6 /(c(\sqrt{12 / c}+1))$ ( $c$ being the central charge of the associated universality class) [19], one obtains the leading term expression

$$
\mathcal{E}(\chi) \sim a+\left(b+b^{\prime} \log \chi\right) / \chi^{\kappa},
$$

with $a, b$ and $b^{\prime}$ coefficients. Remarkably, the validity of this relation is in agreement with numerical simulations with MPSs for a variety of $1 \mathrm{D}$ spin chains, within some accuracy considerations (see Appendix). As illustrated in Fig. 1, the extracted exponent $\kappa$ is very close to the expected value. We suspect that the deviation is due to the fact that (i) the GE converges quickly with $\chi$ to the $\chi=\infty$ value and (ii) the correction is small (as it scales inversely with $\chi$ to some positive power). The larger deviation for smaller $r$ in Fig. 1a and that at larger $h$ in Fig. $1 \mathrm{~b}$ is also due to the fact that the $r=0$ and $h=1$ is not a conformally invariant critical point, and the scaling does not hold. For further discussions, see the Appendix.

\section{Finite-size scaling of GE per site}

Finally, our scaling laws for finite correlation length are in accordance with the finite-size scaling behavior found for 1D systems of size $N$ at the thermodynamic critical point $[12,13,21,22]$. In that case, it was found that the GE per site obeyed the law $\mathcal{E}(N) \sim a+b / N$, with $a$ and $b$ some size-independent coefficients. Up to a logarithmic factor in some cases, this is the same type of behavior that we have found here if the role of the correlation length $\xi$ is now played by the size of the system $N$. In general, this is a manifestation of the well-known property that for a finite system close to criticality, the size of the system plays the role of an infrared cut-off in the correlation length. 


\section{CONCLUSIONS}

Here we have provided a non-trivial lower bound to the GE of MPSs in the thermodynamic limit. We have discussed some sufficient conditions under which this lower bound collapses to an equality, such as space inversion or parity $(\mathrm{P})$ symmetry, in turn justifying the approach considered in previous works to compute the GE. We have also derived scaling laws for the GE per site of infinitesize 1D systems with finite but large correlation length $\xi \gg 1$. These results have also been related to scaling of the GE with the bond dimension of MPSs and the finite-size scaling of the GE for 1D systems.

\section{Acknowledgments}

R. O. acknowledges discussions with J. Fjaerestad, I. P. McCulloch and L. Tagliacozzo, and support from the ARC and UQ. T.-C. W. acknowledges support from NSERC and MITACS.

\section{Appendix A: Further discussions on finite- $\chi$ scaling}

Here we discuss numerical results that further check the validity of Eq. (13). We have performed numerical simulations for the spin-1/2 quantum XY model in the regimes corresponding to both the Ising and XX universality classes (respectively central charges $c=1 / 2$ and $c=1$ ), and for the spin-1/2 antiferromagnetic Heisenberg model $(c=1)$. The MPS approximation to the critical ground state has been obtained using the iTEBD method [20] for $\chi \in[2,40]$, and the GE has been extracted using standard optimization tools.

Before presenting our results, a word is in order regarding potential sources of errors. As opposed to other quantities such as the entanglement entropy or the singlecopy entanglement, the GE at criticality converges fast to a finite value as $\chi$ (the MPS bond dimension) grows. This is indeed a good feature if we are interested in the GE itself in the limit of infinite bond dimension (as opposed to e.g. the entropy, which tends to be divergent). Our fits try to capture the correction to this converged finite value. In the vast majority of the cases, such a correction is small and approaches zero as $\chi \rightarrow \infty$ very quickly. Thus, our fits are sensitive to small numerical errors difficult to control completely. These errors are accentuated if, on top, the GE itself is very small, as is the case of the XY model close to $r=0$ and $h=1$ (where the ground state of the system is separable). If this is the case, then our fits try to capture a tiny correction to a tiny quantity, which may be numerically ill-defined. As we shall see, this seems to be particularly true for the $\mathrm{XY}$ model at $h=1$ and as a function of the anisotropy $r$, where the GE itself is around one order of magnitude smaller than for the XX and Heisenberg models. In turn, this also implies that the our most accurate fit to Eq. (13)

\begin{tabular}{|c|c|c|c|c|}
\hline$r$ & $a$ & $\kappa$ & $b^{\prime}$ & $b$ \\
\hline \hline 0.5000 & 0.015 & $2.76 \pm 0.11$ & $0.010 \pm 0.001$ & $-0.017 \pm 0.002$ \\
0.5625 & 0.017 & $2.50 \pm 0.14$ & $0.012 \pm 0.001$ & $-0.020 \pm 0.002$ \\
0.6250 & 0.019 & $2.37 \pm 0.15$ & $0.013 \pm 0.001$ & $-0.023 \pm 0.002$ \\
0.6875 & 0.021 & $2.18 \pm 0.14$ & $0.014 \pm 0.001$ & $-0.026 \pm 0.002$ \\
0.7500 & 0.023 & $2.07 \pm 0.15$ & $0.014 \pm 0.001$ & $-0.028 \pm 0.003$ \\
0.8125 & 0.024 & $2.00 \pm 0.15$ & $0.015 \pm 0.002$ & $-0.030 \pm 0.003$ \\
0.8750 & 0.026 & $1.93 \pm 0.15$ & $0.016 \pm 0.002$ & $-0.032 \pm 0.003$ \\
0.9375 & 0.028 & $1.89 \pm 0.15$ & $0.017 \pm 0.002$ & $-0.034 \pm 0.004$ \\
1.0000 & 0.029 & $1.85 \pm 0.15$ & $0.017 \pm 0.002$ & $-0.035 \pm 0.004$ \\
\hline
\end{tabular}

TABLE I: Value of the fitted parameters $a, b, b^{\prime}$ and the exponent $\kappa$ together with the associated fitting errors, for the $\mathrm{XY}$ model with $h=1$, for different values of $r$. The fits are to Eq. (13), and in the regime where the GE is not exceedingly small. On average, $\kappa \sim 2.1$. Parameter $a$ has no fitting error since it is extracted directly from our numerical algorithm in the $\chi \rightarrow \infty$ limit.

is obtained for the Heiseiberg model, for which the GE is larger. Nevertheless, and in spite of these considerations, our fits succeed in capturing the essential scaling properties of the different systems with good confidence in some regimes.

Keeping the above considerations in mind, our numerical analysis indicates the following:

(i) For the XY model with $r \neq 0$ and $h=1$ (central charge $c=1 / 2$ universality class), the law in Eq. (13) fits well the GE for $r$ close to 1, whereas for $r$ far from 1 the GE decreases quickly towards very small values. This induces to variations in the different parameters of the fit as a function of $r$, which become stronger as the GE approaches zero (see the data in Table I). The observed variations in the parameters are compatible with the laws $a(r) \sim \alpha_{a}+\beta_{a} r, b(r) \sim-\alpha_{b}-\beta_{b} r, b^{\prime}(r) \sim \alpha_{b^{\prime}}-\beta_{b^{\prime}} / r^{1 / 2}$ and $\kappa(r) \sim \alpha_{\kappa}+\beta_{\kappa} / r^{2}$, for some positive coefficients $\alpha_{a}, \beta_{a}, \alpha_{b}, \beta_{b}, \alpha_{b^{\prime}} \beta_{b^{\prime}}, \alpha_{\kappa}$ and $\beta_{\kappa}$. In spite of this variations, the average behavior of the scaling exponent seems to be $\kappa \sim 2.1$, compatible with the theoretical prediction $\kappa \sim 2$.

(ii) For the XY model with $r=0$ and $0<h<1$ (central charge $c=1$ universality class), we obtain good fits to Eq. (13) in the region close to $h=0$ with values of the scaling exponent around $\kappa \sim 1.2$ (see the data in Table II). Close to $h=1$, where the ground state becomes very little entangled, the GE is again too small to extract the scaling behavior with confidence. Furthermore, the critical point near $h=1$ is not conformally invariant and has dynamical exponent $z=2$, and hence the relation between $\kappa$ and $c$ (the latter being not defined in this regime) does not hold. The observed variations in the parameters are compatible with the laws $a(h) \sim \alpha_{a}-\beta_{a} h^{3}, b(h) \sim-\alpha_{b}+\beta_{b} h^{2}, b^{\prime}(h) \sim \alpha_{b^{\prime}}-\beta_{b^{\prime}} h$ and $\kappa(h) \sim \alpha_{\kappa}+\beta_{\kappa} \exp \left(\gamma_{\kappa} h\right)$, for some positive coefficients $\alpha_{a}, \beta_{a}, \alpha_{b}, \beta_{b}, \alpha_{b^{\prime}} \beta_{b^{\prime}}, \alpha_{\kappa}, \beta_{\kappa}$ and $\gamma_{\kappa}$. Interestingly, we obtain $\beta_{\kappa} \sim 3 \times 10^{-5}$ and $\gamma_{\kappa} \sim 10$, which involves 


\begin{tabular}{|c|c|c|c|c|}
\hline$h$ & $a$ & $\kappa$ & $b^{\prime}$ & $b$ \\
\hline \hline 0.0000 & 0.155 & $1.21 \pm 0.06$ & $0.060 \pm 0.003$ & $-0.171 \pm 0.008$ \\
0.0625 & 0.154 & $1.21 \pm 0.06$ & $0.060 \pm 0.003$ & $-0.171 \pm 0.008$ \\
0.1250 & 0.154 & $1.21 \pm 0.06$ & $0.060 \pm 0.003$ & $-0.170 \pm 0.008$ \\
0.1875 & 0.152 & $1.21 \pm 0.06$ & $0.059 \pm 0.003$ & $-0.168 \pm 0.008$ \\
0.2500 & 0.151 & $1.21 \pm 0.06$ & $0.059 \pm 0.003$ & $-0.167 \pm 0.008$ \\
0.3125 & 0.148 & $1.22 \pm 0.06$ & $0.059 \pm 0.003$ & $-0.166 \pm 0.008$ \\
0.3750 & 0.145 & $1.22 \pm 0.06$ & $0.057 \pm 0.003$ & $-0.162 \pm 0.008$ \\
0.4375 & 0.141 & $1.22 \pm 0.07$ & $0.057 \pm 0.003$ & $-0.159 \pm 0.008$ \\
0.5000 & 0.137 & $1.23 \pm 0.07$ & $0.056 \pm 0.003$ & $-0.156 \pm 0.009$ \\
0.5625 & 0.132 & $1.23 \pm 0.07$ & $0.055 \pm 0.003$ & $-0.152 \pm 0.009$ \\
0.6250 & 0.125 & $1.25 \pm 0.08$ & $0.054 \pm 0.004$ & $-0.146 \pm 0.009$ \\
0.6875 & 0.118 & $1.29 \pm 0.09$ & $0.054 \pm 0.004$ & $-0.142 \pm 0.010$ \\
0.7500 & 0.108 & $1.33 \pm 0.10$ & $0.053 \pm 0.005$ & $-0.136 \pm 0.011$ \\
0.8125 & 0.097 & $1.40 \pm 0.12$ & $0.051 \pm 0.006$ & $-0.128 \pm 0.012$ \\
0.8750 & 0.081 & $1.64 \pm 0.21$ & $0.061 \pm 0.011$ & $-0.133 \pm 0.021$ \\
\hline
\end{tabular}

TABLE II: Value of the fitted parameters $a, b, b^{\prime}$ and the exponent $\kappa$ together with the associated fitting errors, for the XX model for different values of $h$. The fits are to Eq. (13), and in the regime where the GE is not exceedingly small. On average, $\kappa \sim 1.3$. Parameter $a$ has no fitting error since it is extracted directly from our numerical algorithm in the $\chi \rightarrow \infty$ limit. a smooth behavior of the exponent for low values of $h$, with a rapid increase for large $h$. Despite of this variations, the average scaling exponent that we obtain in this region is $\kappa \sim 1.3$, compatible with the theoretical result (which is also $\kappa \sim 1.3$ ).

(iii) For the spin-1/2 Heisenberg model (central charge $c=1$ universality class), our fit to Eq. (13) seems to indicate that $a \sim 0.259, b \sim-0.259 \pm 0.004, b^{\prime} \sim 0.078 \pm 0.010$ and $\kappa \sim 1.25 \pm 0.08$, compatible also with the theoretical result $\kappa \sim 1.3$.

Thus, our data seems compatible with the scaling law in Eq. (13) with values of the scaling exponent $\kappa$ not too far from the theoretical predictions for conformallyinvariant quantum critical points, within the considerations of numerical accuracy that we discussed previously. Let us also remark that, independently of the specific form of scaling, the fact that the GE converges quickly with $\chi$ to a specific value is also a remarkable and useful property, as e.g. the infinite- $\chi$ limit can be easily extracted.
[1] S. Sachdev, Quantum Phase Transitions (Cambridge University Press, Cambridge, 1999).

[2] G. Vidal , J. I. Latorre, E. Rico, and A. Kitaev, Phys. Rev. Lett. 90227902 (2003); J. I. Latorre, E. Rico, G. Vidal, Quantum Inf. and Comput. 4, 48 (2004). V. Korepin, Phys. Rev. Lett. 92096402 (2004); A. R. Its, B. Q. Jin, and V. E. Korepin, J. Phys. A: Math. Gen. 38, 29752990, (2005); P. Calabrese, J. Cardy, JSTAT 0406:002 (2004); J. Eisert, M. Cramer, Phys. Rev. A 72, 042112 (2005); I. Peschel, J. Zhao, JSTAT P11002 (2005); R. Orús, J. I. Latorre, J. Eisert, and M. Cramer, Phys. Rev. A 73, 060303(R) (2006); A. Riera, J. I. Latorre, Phys. Rev. A 74052326 (2006).

[3] K. Wilson, J. Kogut, Phys. Rep. 12C, 75 (1974); K. Wilson, Rev. Mod. Phys. 47773 (1975); A. B. Zamolodchikov, JETP Lett. 43730 (1986); F. Verstraete, J. I. Cirac, J. I. Latorre, E. Rico, and M. M. Wolf, Phys. Rev. Lett. 94, 140601 (2005); J.I. Latorre, C.A. Lutken, E. Rico, G. Vidal, Phys. Rev. A 71, 034301(2005); R. Orús, Phys.Rev. A 71, 052327 (2005); ibid 73, 019904 (2006).

[4] See e.g. S. Bose, Con. Phys. 48, Issue 1, 13 - 30 (2007).

[5] G. Vidal, Phys. Rev. Lett. 91, 147902 (2003); D. Aharonov, D. Gottesman, S. Irani, and J. Kempe, Comm. Math. Physics, vol. 287, 41 (2009); N. Schuch, J. I. Cirac, arXiv:0910.4264.

[6] S. R. White, Phys. Rev. Lett. 69, 2863 (1992); S. R. White, Phys. Rev. B 48, 10345 (1992); U. Schollwoeck, Rev. Mod. Phys. 77, 259 (2005).

[7] I. Affleck, T. Kennedy, E. H. Lieb, and H. Tasaki, Phys. Rev. Lett. 59, 799 (1987); I. Affleck, T. Kennedy, E. H. Lieb, and H. Tasaki, Commun. Math. Phys. 115, 477
(1988); S. Ostlund, S. Rommer, Phys. Rev. Lett. 75, 3537 (1995); M. Fannes, B. Nachtergaele, R. F. Werner, Commun. Math. Phys. 144, 443 (1992); D. Perez-García, F. Verstraete, M.M. Wolf, and J.I. Cirac, Quantum Inf. Comput. 7, 401 (2007).

[8] F. Verstraete, J. I. Cirac, Phys. Rev. B 73, 094423 (2006); M. Hastings, JSTAT, P08024 (2007); M. Hastings, Phys. Rev. B 76, 035114 (2007); M. Hastings, Phys. Rev. B 73, 085115 (2006);

[9] See e.g. L. Amico, R. Fazio, A. Osterloh, and V. Vedral, Rev. Mod. Phys. 80, 517 (2008).

[10] T.-C. Wei, P. M. Goldbart, Phys. Rev. A 68, 042307 (2003).

[11] T.-C. Wei, D. Das, S. Mukhopadyay, S. Vishveshwara, and P. M. Goldbart, Phys. Rev. A 71, 060305(R) (2005).

[12] T.-C. Wei, Phys. Rev. A 81, 062313 (2010); R.Orús, Phys. Rev. Lett. 100, 130502 (2008); A. Botero, B. Reznik, arXiv:0708.3391; R. Orús, Phys. Rev. A 78, 062332 (2008); R. Orús and T.-C. Wei, Phys. Rev. B 82, 155120 (2010).

[13] Q.-Q. Shi, R. Orús, J. O. Fjærestad, and H.-Q Zhou, New J. Phys. 12, 025008 (2010).

[14] Our results can also be generalized to these cases, for example, by considering the GE per block [12].

[15] For most cases this turns out to be a correct assumption. However, it may be possible to find cases where this does not hold.

[16] C.-Y. Huang, F.-L. Lin Phys. Rev. A 81, 032304 (2010).

[17] S. Tamaryan, T.-C. Wei, D. Park, Phys. Rev. A 80, 052315 (2009); R. Hübener, M. Kleinmann, T.-C. Wei, C. González-Guillén, and O. Gühne, Phys. Rev. A 80, 
032324 (2009); M. Hayashi, D. Markham, M. Murao, M. Owari, and S.Virmani, J. Math. Phys. 50, 122104 (2009); R. Orús, S. Dusuel, J. Vidal, Phys. Rev. Lett. 101, 025701 (2008).

[18] We thank Carl Bender for pointing out the connection. See, e.g., C. M. Bender, Rep. on Prog. in Phys. 70, 947 (2007); A. Guo, G. J. Salamo, D. Duchesne, R. Morandotti, M. Volatier-Ravat, V. Aimez, G. A. Siviloglou and D. N. Christodoulides, Phys. Rev. Lett. 103, 093902 (2009); C. E. Rüter, K. G. Makris, R. El-Ganainy, D. N. Christodoulides, M. Segev, and D. Kip, Nat. Phys. 6, 192 (2010);

[19] L. Tagliacozzo, T. R. de Oliveira, S. Iblisdir, and J. I. Latorre, Phys. Rev. B 78, 024410 (2008); F. Pollmann, S. Mukerjee, A. M. Turner, and J. E. Moore, Phys. Rev. Lett. 102, 255701 (2009).

[20] I. P. McCulloch, arXiv:0804.2509; G. Vidal, Phys. Rev.
Lett. 98, 070201 (2007); R. Orús, G. Vidal, Phys. Rev. B 78, 155117 (2008).

[21] T.-C. Wei, S. Vishveshwara, and P. M. Goldbart, Quantum Inf. Comput. 11, 0326 (2011).

[22] J.-M. Stéphan, G. Misguich, and F. Alet, Phys. Rev. B 82, 180406(R) (2010).

[23] R. Bhatia, Matrix analysis (Springer-Verlag, NY) 1997.

[24] I. P. McCulloch, private communication.

[25] For example, see M. Asoudeh, V. Karimipour, A. Sadrolashrafi, Phys. Rev. B 75, 224427 (2007). Notice that a similar consideration leads to the condition for time reversal $(\mathrm{T})$ symmetry in terms of MPS: $A^{s *}=$ $V A^{(-s)} V^{-1}$, for some invertible $V$, and $-s$ is the index corresponding to that of the time reversed state of $s$.

[26] The results of this paper can be easily generalized to other periodicities of translational invariance. 